Please do not remove this page

RMIT

UNIVERSITY

\title{
Joint time-domain/frequency-domain impulsive noise reduction in OFDM-based power line communications
}

Al Mawali, Khalifa; Sadik, Amin; Hussain, Zahir

https://researchrepository.rmit.edu.au/esploro/outputs/9921863932401341/filesAndLinks?institution=61RMIT_INST\&index=null

Al Mawali, K., Sadik, A., \& Hussain, Z. (2008). Joint time-domain/frequency-domain impulsive noise reduction in OFDM-based power line communications. Australasian Telecommunications Networks and Applications Conference 2008 (ATNAC-2008), 138-142. https://doi.org/10.1109/ATNAC.2008.4783311

Published Version: https://doi.org/10.1109/ATNAC.2008.4783311

Repository homepage: https://researchrepository.rmit.edu.au

(c) 2008 IEEE. Personal use of this material is permitted. However, permission to reprint/republish this material for advertising or promotional purposes or for creating new collective works for resale or redistribution to servers or lists, or to reuse any copyrighted component of this work in other works must be obtained from the IEEE.

Downloaded On 2023/04/26 21:40:28 +1000 


\title{
Joint Time-domain/Frequency-domain Impulsive Noise Reduction in OFDM-based Power Line Communications
}

\author{
Khalifa Al-Mawali, Amin Z. Sadik, and Zahir M. Hussain \\ School of Electrical \& Computer Engineering, RMIT University, Melbourne, Australia \\ k.almawali@student.rmit.edu.au, amin.sadik@rmit.edu.au,zmhussain@ieee.org
}

\begin{abstract}
Impulsive noise is one of the major challenges in power line communications and can cause serious problems in OFDM-based PLC systems. In this paper, we propose a combined Time-domain/Frequency-domain technique for impulsive noise reduction in OFDM-based PLC systems. The performance of the proposed technique is studied against well known time-domain nonlinearities by means of computer simulations. The obtained simulation results show that the Combined TD/FD technique performs better than practically used nonlinearities and can reduce the adverse effect of impulsive noise significantly.
\end{abstract}

-Keywords: Power line Communications, Impulsive noise, OFDM.

\section{INTRODUCTION}

During the last decade, a great deal of attention has been directed towards the use of electric power lines for multimedia communications. Power Line Communications (PLC) offers the advantage of using the existing and widespread power distribution infrastructure to provide home networking as well as broadband services. However, due to hostile channel characteristics, communication over power lines faces serious challenges including noise, attenuation and multipath propagation [1].

Noise in power lines forms a significant dilemma for data transmission. This is because it can not be described by Additive white Gaussian Noise (AWGN) as the case in many other communication channels [2]. Noise in power line environment can be classified into five types [3]. These are coloured background noise, narrow-band noise, periodic impulsive noise asynchronous to the mains frequency, impulsive noise synchronous to the mains frequency and asynchronous impulsive noise. The first three types of noise usually remain stationary over long periods of time (seconds, minutes or hours) and can be summarised as background noise. The last two types are time-variant and can be summarised as impulsive noise. Impulsive noise is mainly caused by power supplies (synchronous to mains frequency) or by switching transients in the network (Asynchronous to the mains power). It has a random occurrence and its duration varies from few microseconds to milliseconds. Practical experiments in power lines [3] show that the PSD of impulsive noise exceeds the PSD of background noise by minimum of 10-15 $\mathrm{dB}$ and may sometimes reach $50 \mathrm{~dB}$.

The harsh channel environment of power lines requires a transmission scheme that can effectively cope with hostile channel conditions. Due to its robustness to multipath, selective fading and different kinds of interference, Orthogonal Frequency Division Multiplexing (OFDM) is a major candidate for PLC systems. OFDM is a mature multicarrier transmission technique and have been adopted in several wideband digital communication systems. Examples of its applications include Digital Audio Broadcasting (DAB), Terrestrial digital TV (DVB-T), wireless LANs and Wi-Max. OFDM performs better than single carrier in the presence of impulsive noise [1]. This is due to the fact that OFDM spreads the effect of impulsive noise over multiple symbols due to Discrete Fourier Transform (DFT) algorithm. Moreover, the addition of Cyclic Prefix (CP) in OFDM symbols can mitigate the effect of multipath.

Despite the superiority of OFDM in the presence of impulsive noise, it is necessary to employ mitigation techniques in order to further reduce its effect in data transmission. A simple technique of impulsive noise mitigation is to precede OFDM receiver with a memoryless nonlinearity. This method has been addressed for conventional OFDM receivers in wireless applications [7]. However, power line channels exhibit different channel characteristics to other communication channels. In this paper, we adopt a practically proven PLC channel model [3],[4] to compare the performance of three memoryless nonlinearity methods (Clipping, Blanking and Clipping/Blanking) for mitigation of impulsive noise in OFDM systems over PLC channel. We also propose a combined time-domain/frequencydomain technique for impulsive noise reduction in OFDMbased PLC systems.

\section{System Model}

\section{A. OFDM System}

Orthogonal frequency Division Multiplexing (OFDM) is a very well known multi-carrier transmission technique that can cope well with PLC channel conditions. In OFDM systems a high-speed serial data stream is split into a number of parallel slow data streams that are carried in multiple orthogonal subcarriers by means of Inverse Discrete Fourier Transform 
(IDFT). The discrete time OFDM signal can be expressed as:

$$
s(n)=\frac{1}{\sqrt{N}} \sum_{k=0}^{N-1} S_{k} e^{j 2 \pi \frac{k}{N} n}
$$

Where $N$ is the number of sub-carriers, $S_{k}$ is a sequence of QAM synbols. In order to combat iner-channel interference (ICI) and inter-symbol interference (ISI), OFDM uses a cyclic prefix $(\mathrm{CP})$ that is appended at the start of OFDM symbols.

\section{B. Channel Model}

Originally, the power line network was not specifically designed for data transmission and provides a harsh environment for it [2]. It differs significantly in topology, structure, and physical properties from conventional communication channels such as twisted pair, coaxial, or fiber-optic cables [4]. The most influencing properties of power lines in the performance of high speed communications are signal distortion due to frequency-dependant cable loses, multi-path propagation and noise [3]. These factors and others have to be taken into account in order for a successful communication over power lines. As a result, the need for a realistic and practical model for the power line channel transfer characteristics is inevitable. Zimmermann [4] has proposed such a practical channel model that is suitable for describing the transmission behaviour of power line channels. His model is based on practical measurements of actual power line networks. This power line channel model is expressed by the channel transfer function:

$$
H(f)=\sum_{i=1}^{N_{p}} \underbrace{c_{i}}_{\begin{array}{c}
\text { weighting } \\
\text { factor }
\end{array}} \cdot \underbrace{e^{-\left(a_{0}+a_{1} f^{k}\right) d_{i}}}_{\begin{array}{c}
\text { attenuation } \\
\text { portion }
\end{array}} \cdot \underbrace{e^{-j 2 \pi f\left(d_{i} / v p\right)}}_{\begin{array}{c}
\text { delay } \\
\text { portion }
\end{array}}
$$

Where $N_{p}$ is the number of multipaths, $c_{i}$ and $d_{i}$ are the weighting factor and length of the ith path respectively. Frequency-dependant attenuation is modelled by the parameters $a_{0}, a_{1}$ and $k$. In the model, the first exponential presents attenuation in the PLC channel, whereas the second exponential, with the propagation speed $v p$, describes the echo scenario. The attenuation parameters for 15-path model were obtained using physical measurements [4]. In this paper we use this widely proven model to simulate our proposed algorithm and compare its performance against nonlinearity methods in mitigating the impulsive noise encountered in practical PLC systems.

\section{Impulsive Noise}

In contrast to most other communication channels, the noise in power line channels can not be described as Additive White Gaussian Noise (AWGN). In PLC environments, data transmission may encounter different types of noise. These are coloured background noise, narrow-band noise, periodic impulsive noise asynchronous to the mains frequency, impulsive noise synchronous to the mains frequency and asynchronous impulsive noise. These five types of noise can be summarised into two major types: background noise and impulsive noise.
To analyse their effect in OFDM-based PLC systems, the background noise $\left(w_{k}\right)$ is modelled as AWGN with mean zero and variance $\sigma_{w}^{2}$, and the impulsive noise $\left(i_{k}\right)$ is given by:

$$
i_{k}=b_{k} g_{k}
$$

Where $b_{k}$ is the Poisson Process which is the arrival of impulsive noise, and $g_{k}$ is white Gaussian process with mean zero and variance $\sigma_{i}^{2}$. This means that the arrival of impulsive noise follows a Poisson distribution with a rate $\lambda$ units per second, so that the probability of event of $\mathrm{k}$ arrivals in $\mathrm{t}$ seconds is:

$$
P(k)=P(X=k)=e^{-\lambda} \frac{\lambda^{k}}{k !}, \quad k=0,1,2, \ldots
$$

The amplitude of impulsive noise, on the other hand, follows Gaussian distribution. Let $s_{k}$ be the transmitted OFDM signal as in (1). The received signal after down-conversion, analogto-digital conversion assuming perfect synchronization can be represented as:

$$
r_{k}=s_{k} * h+w_{k}+i_{k} \quad k=0,1,2, \ldots, N-1
$$

where $h$ is the PLC channel impulse response which is the Inverse Fourier Transform of the transfer function defined in (2). The symbol $*$ denotes convolution. Figure 1 shows the total noise resulting from AWGN and impulsive noise

\section{Proposed Technique for Impulsive Noise Mitigation}

In OFDM systems, the long symbol period gives the signal more robustness to impulsive noise, since the impulse noise energy is spread over the subcarriers. Nevertheless, if mitigation techniques are not employed, impulsive noise can still have a significant effect in the performance of OFDM systems, especially in a medium like power lines. Different techniques for impulsive noise mitigation have been discussed in the literature [3][5][6] . Due to their simplicity, nonlinearity techniques are often used in practical applications for impulsive noise mitigation. An overview of three simple nonlinearity techniques including clipping, blanking and clipping/blanking is given in the following subsection. A frequency-domain technique for impulsive noise suppression that was proposed by Zhidkov [6] is also explained briefly. Our proposed technique for impulsive noise mitigation in OFDM systems combines time-domain techniques with the above mentioned frequencydomain mitigation technique. Simulation results show that the proposed techniques performs better than the previous techniques in terms of BER reduction.

\section{A. Time domain Methods}

Practical applications often use the following nonlinearity techniques for impulsive noise suppression [7]. These techniques change only the amplitude of the signal according to a specific threshold without changing the phase. They are employed at the time domain signal before the demodulation process using Fast Fourier Transform (FFT). 


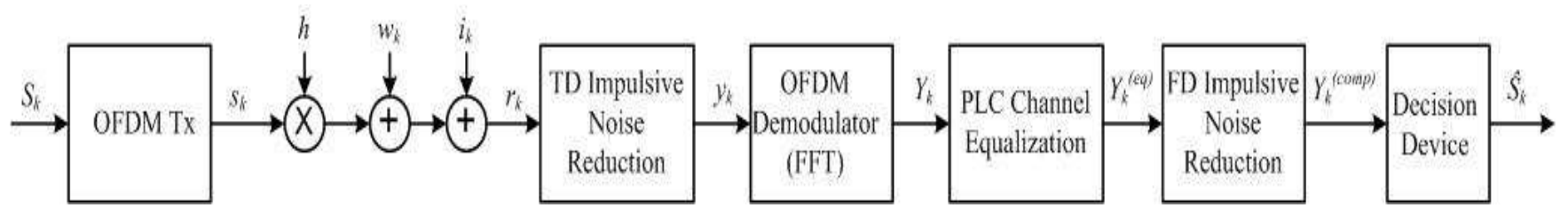

Fig. 1. Block diagram of the OFDM-based PLC system with impulsive noise reduction

1) Clipping

$$
y_{k}=\left\{\begin{array}{ll}
r_{k}, & \left|r_{k}\right| \leq T_{c} \\
T_{c} e^{j a r g\left(r_{k}\right)}, & \left|r_{k}\right|>T_{c}
\end{array}, \quad k=0,1, \ldots, N-1\right.
$$

where $T_{c}$ is the Clipping Threshold.

2) Blanking

$$
y_{k}=\left\{\begin{array}{ll}
r_{k}, & \left|r_{k}\right| \leq T_{b} \\
0, & \left|r_{k}\right|>T_{b}
\end{array}, \quad k=0,1, \ldots, N-1\right.
$$

Where $T_{b}$ is the blanking threshold.

3) Clipping / Blanking

$$
y_{k}=\left\{\begin{array}{ll}
r_{k}, & \left|r_{k}\right| \leq T_{1} \\
T_{1} e^{j a r g\left(r_{k}\right)}, & T_{1}<\left|r_{k}\right| \leq T_{2} \\
0, & \left|r_{k}\right|>\leq T_{2}
\end{array}, k=0,1, \ldots, N-1\right.
$$

Where the clipping threshold $\left(T_{c}\right)$ is smaller than the blanking threshold $\left(T_{b}\right)$.

\section{B. Frequency-domain suppression method}

A suppression technique of impulsive noise in OFDM receivers was proposed in [6]. The technique is based on a frequency-domain approach where impulsive noise is compensated for after OFDM demodulation using (FFT). In this section we provide a brief overview about this technique. More details are available in [6]. In the receiver side of OFDM system, the signal in (5) can be expressed, after channel equalization and DFT demodulation, as:

$$
R_{k}^{(e q)}=S_{k}+W_{k} \hat{H}_{k}^{-1}+I_{k} \hat{H}_{k}^{-1}, \quad k=0,1, \ldots, N-1
$$

Where $H$ is the channel transfer function, $W$ and $U$ are the DFT of the AWGN and impulsive noise respectively. The main idea of the algorith is to get an estimation of the impulsive noise term $I_{k} \hat{H}_{k}^{-1}$ and subtract it from the equalizer output. To do this, a preliminary estimate of the transmitted beseband symbol has to be obtained. this is done through demapping the data subcarriers to nearest constellation points, setting silent subcarriers to zero and replacing the pilot subcarriers by their known values. By rearranging (9), estimation of the total noise term $D_{k}=W_{k}+I_{k}$ can be found by the following expression:

$$
\hat{D}_{k}=\hat{H}_{k}\left(R_{k}^{(e q)}-\hat{S}_{k}\right), \quad k=0,1, \ldots, N-1
$$

The total noise term $D_{k}$ is then transformed into the time domain by means of IDFT and a peak detector is used to obtain the estimated impulsive noise signal $\hat{i}_{k}$ in the time domain.
In a final step, the DFT of $\hat{i}_{k}$ is computed and divided by the channel transfer function. The signal after impulsive noise reduction can be attained according to the following:

$$
R_{k}^{(c o m p)}=R_{k}^{(e q)}-\hat{I}_{k} \hat{H}_{k}^{-1}, \quad k=0,1, \ldots, N-1
$$

\section{COMbined TIME-DOMAIN FREQUENCY-DOMAIN FOR IMPULSIVE NOISE REDUCTION}

For high data rate communications over PLC mediums, more reduction of impulsive noise is necessary. In this paper, we propose a joint time-domain/frequency-domain technique for impulsive noise reduction in OFDM-based PLC systems. Figure 1 shows how this technique is implemented in OFDM systems. The impulsive noise in received OFDM symbols $r_{k}$ is first reduced using a time domain preprocessor. The preprocessor is made of a Clipping/Blanking nonlinearity circuit. This combined nonlinearity technique is known to perform better than clipping and blanking nonlinearities [7]. Our simulation results in the following section of this paper confirm this result. In order to further improve the impulsive noise mitigation, a frequency-domain suppression technique is applied to the OFDM signal after channel equalization and demodulation by means of DFT. Our simulation results show a significant improvement in Bit Error Rate as compared to conventional OFDM systems and also OFDM-systems with nonlinearity-based impulsive noise reduction.

\section{EXPERIMENTAL RESULTS AND DISCUSSION}

Matlab software was utilized to study the performance of the proposed combined Time-Domain/Frequency-Domain impulsive noise mitigation technique. A random signal is mapped into QPSK symbols and modulated using OFDM with 128 subcarriers and passed through a PLC multipath channel with 15 paths. The arrival of impulsive noise is assumed to follow a Poisson distribution and is added to the signal as well as AWGN. Fig. (2) shows impulsive noise samples as well as the background AWGN.

Different impulsive noise reduction techniques are then employed in the OFDM receiver and their performance is compared. SNR in all figures is defined according to the following calculations:

Let $p_{n}$ be the total noise power resulting from AWGN and impulsive noise, and $p_{x}$ is the average PSK or QAM symbol power. The signal-to-noise (SNR) ratio can be defined as:

$$
S N R=\frac{p_{x}}{p_{n}}=\frac{p_{x}}{\sigma_{w}^{2}+p_{i}}
$$




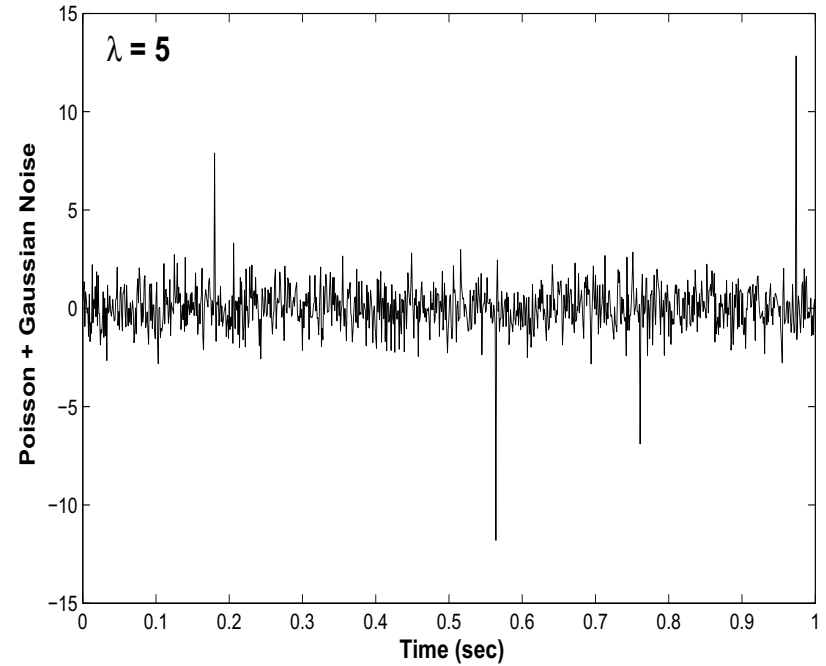

Fig. 2. Impulsive noise added to Additive White Gaussian Noise

where $\sigma_{w}^{2}$ and $p_{i}$ are the mean AWGN power and mean impulsive noise power. Since impulsive noise arrival is modelled by Poisson process with Poisson parameter $\lambda$, the following expression can be obtained:

$$
S N R=\frac{p_{x}}{\sigma_{w}^{2}+(1 / \lambda) \sigma_{i}^{2}}
$$

where $\sigma_{i}^{2}$ is the mean impulse power and is modelled by Gaussian process as indicated in (3). A ratio $r$ between impulsive noise power and background noise power (AWGN) is defined as $r=p_{i} / \sigma_{w}^{2}$. In our simulations we use $\lambda=5$ and $r=1.5$.

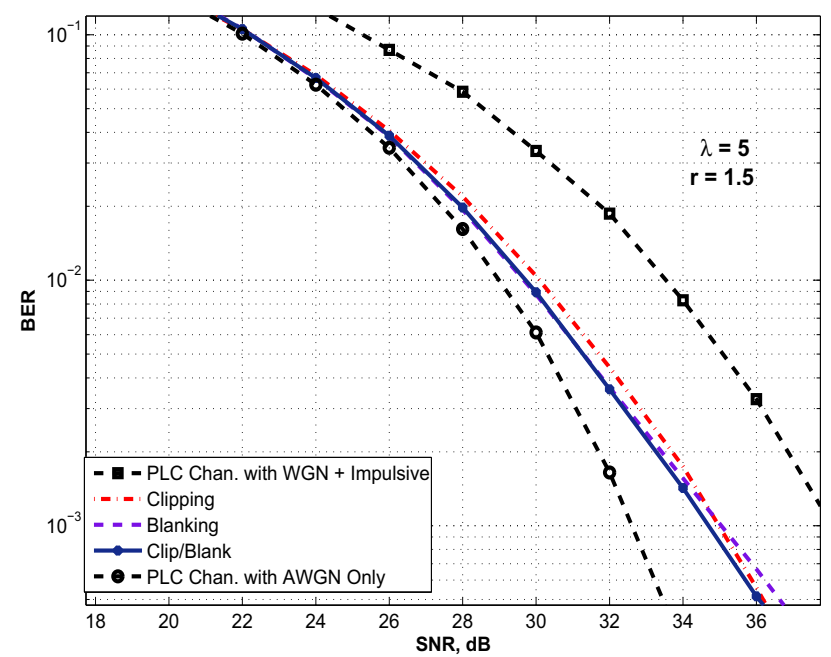

Fig. 3. A comparison of time domain nonlinearity techniques for impulsive noise reduction

Fig. (3) illustrates a comparison between the three simple nonlinearity techniques used for inpulsive noise suppression. It can clearly be noticed that blanking nonlinearity performs better than clipping. It can also be observed from the figure that the best nonlinearity solution to impulsive noise is the combined clipping/blanking.

The superior performance of the proposed combined Timedomain/Frequency-domain impulsive noise suppression technique can be observed from fig.(4). It is obviously seen that the proposed technique outperforms the clipping/blanking technique by more than $5 \mathrm{~dB}$ at high SNR. The proposed technique achieves BER performance close to that of that of signal with only AWGN, which means that the effect of impulsive noise is almost eliminated.

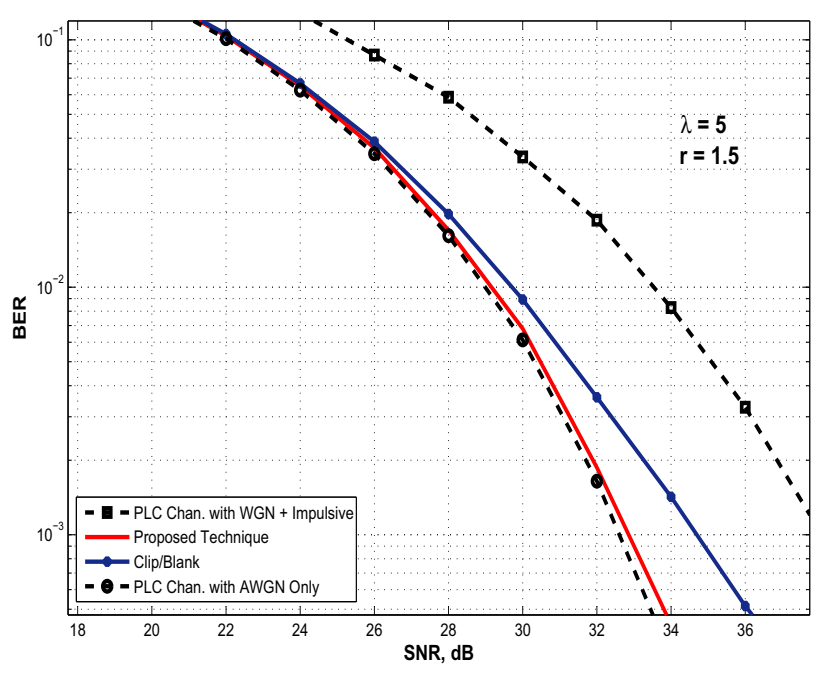

Fig. 4. Performance comparison of combined TD/FD technique and Clipping/Blanking nonlinearity for OFDM-Based PLC

\section{Vi. Conclusion}

Impulsive noise is one of the major challenges in power line communications and can cause serious problems in OFDM-based PLC systems. In this paper, we proposed a combined Time-domain/Frequency-domain technique for impulsive noise reduction in OFDM-based PLC systems. The performance of the proposed technique is studied against well known time-domain nonlinearities by means of computer simulations. The obtained simulation results show that the Combined TD/FD technique performs better than practically used nonlinearities and can reduce the adverse effect of impulsive noise significantly.

\section{REFERENCES}

[1] Y. H. Ma, P. L. So and E.Gunawan, "Performance analysis of OFDM systems for broadband power line communications under impulsive noise and multipath," IEEE Trans. Power Delivery, vol. 20, No. 2, pp. 674-681, April 2005.

[2] M. S Yousuf, and M. El-Shafei, "Power line communications: an overview - part 1,' Innovations '07, pp. 218-222. 
[3] M. Zimmermann and K. Dostert, "Analysis and modeling of impulsive noise in broad-band powerline communications," IEEE Trans. Electromagn. Compat., vol. 44, No. 1, pp. 249-258, Feb. 2002.

[4] M. Zimmermann and K. Dostert, "A multipath model for the pwerline channel," IEEE Trans. Communications, vol. 50, No. 4, pp. 553-559, April. 2002.

[5] F Abdelkefi, P. Duhamel, and F. Alberge, "Impulsive noise cancellation in multicarrier transmission," IEEE Trans. Communications, vol. 53, No. 1, pp. 94-106, Jan. 2005.

[6] S. Zhidkov, "Inpulsive noise suppression in OFDM based communication systems," IEEE Trans. Consumer Electronics, vol. 49, No. 4, pp. 944-948, Nov. 2003.

[7] S. Zhidkov, "Analysis and comparison of several simple impulsive noise mitigation schemes for OFDM receivers," IEEE Trans. Communications, vol. 56, No. 1, pp. 5-9, Jan. 2008.

[8] M. Ghosh,"Analysis of the effect of impulsive noise on multicarrier and single carrier QAM systems," IEEE Trans. Communications, vol. 44, No. 2, pp. 145-147, Feb. 1996.

[9] S. Zhidkov, "Performance analysis and optimization of OFDm receiver with blanking nonlinearity in inpulsive noise environment," IEEE Trans. Vehicular Technology, vol. 55, No. 1, pp. 234-238, Jan. 2006.

[10] M. Gotz, M. Rapp, and K. Dostert, "Power line channel characteristics and their effect on communication system design," IEEE Communications Magazine, vol. 42, issue 4, pp. 78-86, April. 2004. 